\title{
NILPOTENT AUTOMORPHISM GROUPS OF BORDERED KLEIN SURFACES
}

\author{
COY L. MAY
}

\begin{abstract}
Let $X$ be a compact bordered Klein surface of algebraic genus $g \geqslant 2$, and let $G$ be a group of automorphisms of $X$. Then the order of $G$ is at most $12(g-1)$. Here we improve this general bound in an important special case. We show that if $G$ is nilpotent, then the order of $G$ is at most $8(g-1)$. This bound is the best possible. We construct infinite families of surfaces that have a nilpotent automorphism group of order $8(g-1)$. The nilpotent groups of maximum possible order must be 2-groups. We prove that if the nilpotent group $G$ acts on a bordered surface of genus $g$ such that $o(G)=8(g-1)$, then $g-1$ is a power of 2 . Further, our examples show that for each nonnegative integer $t$ there is a bordered surface with genus $g=2^{t}+1$ and a group of automorphisms of order $8(g-1)$.
\end{abstract}

1. Introduction. Let $X$ be a compact bordered Klein surface of algebraic genus $g \geqslant 2$. Then the automorphism group $G$ of $X$ is finite, and the order of $G$ is at most $12(g-1)$ [3]. If the order of $G$ is the largest possible, then $G$ is called an $M^{*}$-group. $M^{*}$-groups were introduced in [4] and have been studied in several recent papers ([2, 5, 7], among others).

There is certainly a wealth of $M^{*}$-groups. These groups may be solvable as well as nonsolvable, and infinitely many examples of each type are known. Many important finite groups are $M^{*}$-groups. For example, $\operatorname{PSL}(2, p)$ is an $M^{*}$-group if $p$ is any odd prime except 3, 7, and 11 [7].

$M^{*}$-groups can even be supersolvable. These especially tractable groups were examined in [5]. Among the $M^{*}$-groups the supersolvable ones are completely determined by their order. An $M^{*}$-group $G$ is supersolvable if and only if $o(G)=4$ - $3^{r}$ for some positive integer $r$ [5, Theorem 1]. Further there is an $M^{*}$-group of order $4 \cdot 3^{r}$ for each $r$ [5, Theorem 2].

However, none of the supersolvable $M^{*}$-groups can be nilpotent [5, §3]. A natural task, then, is to improve the bound $12(g-1)$ for the size of a nilpotent automorphism group of a bordered Klein surface. This is not hard to do. We show that if $G$ is a nilpotent group of automorphisms of a bordered Klein surface of genus $g \geqslant 2$, then the order of $G$ is at most $8(g-1)$. This bound is the best possible. We present several examples of surfaces of low genus that have a nilpotent automorphism group

Received by the editors February 28, 1986 and, in revised form, July 21, 1986.

1980 Mathematics Subject Classification (1985 Revision). Primary 30F10; Secondary 14H30.

Key words and phrases. Bordered Klein surface, genus, automorphism, nilpotent group, $M^{*}$-group, full covering 
of order $8(g-1)$. We also construct some infinite families of surfaces for which this bound is attained. Interestingly, though, only one surface among our examples is nonorientable.

We also examine the nilpotent groups of maximum possible order. These groups must be 2-groups. We prove that if the nilpotent group $G$ acts on a bordered surface of genus $g$ such that $o(G)=8(g-1)$, then $g-1$ is a power of 2 . Further, our examples show that for each nonnegative integer $t$ there is a bordered surface with genus $g=2^{t}+1$ and a group of automorphisms of order $8(g-1)$; as a 2-group, the group is of course nilpotent.

The corresponding results for Riemann surfaces and (orientation-preserving) groups of automorphisms have recently been obtained by Zomorrodian [8]. If $G$ is a nilpotent group of automorphisms of a Riemann surface $M$ of genus $g \geqslant 2$, then $o(G) \leqslant 16(g-1)[8$, p. 252]. The groups of largest possible order are also 2-groups, and if $g-1$ is a power of 2 , then there is a Riemann surface of genus $g$ with an automorphism group of order $16(g-1)$ [8, p. 253].

Not surprisingly, our results for bordered Klein surfaces are similar to those of Zomorrodian [8], even though we do not rely on his work. There is an important difference, however. Hurwitz's bound for the size of the automorphism group of a Riemann surface is $84(g-1)$. But these Hurwitz groups of order $84(g-1)$ cannot be solvable, so that, as expected, Zomorrodian's bound for the size of a nilpotent group is much smaller than $84(g-1)$. This is in marked contrast with the results for bordered surfaces. Since $M^{*}$-groups can be supersolvable, the bound $8(g-1)$ is not far from the general bound $12(g-1)$.

2. Automorphism groups and quotient spaces. We assume that all surfaces are compact. For any Klein surface $X$, let $A(X)$ denote the group of automorphisms of $X$.

Let $X$ be a bordered Klein surface of (algebraic) genus $g \geqslant 2$. Topologically the surface $X$ is characterized by orientability, the number $k$ of boundary components, and its topological genus $p$. Then the genus $g$ is given by the following important relation:

$$
g= \begin{cases}2 p+k-1 & \text { if } X \text { is orientable, } \\ p+k-1 & \text { if } X \text { is nonorientable }\end{cases}
$$

Now let the finite group $G$ of order $r$ act on $X$, that is, let $G$ be a subgroup of $A(X)$. Then the quotient space $Y=X / G$ is a bordered Klein surface, and the quotient map $\pi: X \rightarrow Y$ is a ramified $r$-sheeted covering (with folding) of $Y$. The quotient map $\pi$ is ramified at a finite number of points of $X$. For more details, see [3, pp. 200, 201]. Suppose $k$ is the ramification index of $\pi$ at some point of $X$. It is basic that $k$ divides $o(G)$, since $k$ is the order of a subgroup of $G[3$, p. 203].

Let $\gamma$ denote the genus of the quotient space $Y$. The genus of $X$ can be calculated from $\gamma$ and the ramification indices of the covering by using the Riemann-Hurwitz formula for coverings of bordered surfaces [3, p. 201]. In particular, if $\pi$ is unramified,

$$
2 g-2=r(2 \gamma-2)
$$


The Riemann-Hurwitz formula was used in [3] to establish the general bound $12(g-1)$ for the order of an automorphism group. If the order of the group is large enough, then the group's action is also determined. Here we need the following result about automorphism groups of the second largest possible order.

THEOREM A [3, p. 207]. Let $G$ be a group of automorphisms of a bordered Klein surface $X$ of genus $g \geqslant 2$. If $o(G)<12(g-1)$, then $o(G) \leqslant 8(g-1)$. If $o(G)=$ $8(g-1)$, then the quotient space $X / G$ is the disc $D$ and the quotient map $\pi: X \rightarrow D$ is ramified over exactly 4 points of $D$, all on $\partial D$, with ramification indices $2,2,2,4$.

The analogous result about the action of an $M^{*}$-group is stated explicitly in [ 2 , Theorem B, p. 267]. Groups of order $8(g-1)$ will be important here. There is a nice correspondence between regular maps of type $\{4, q\}$ and bordered Klein surfaces of genus $g \geqslant 2$ with $8(g-1)$ automorphisms [2, p. 282].

3. The main result. The first important result about $M^{*}$-groups was that they must have a certain partial presentation $\left[4\right.$, p. 5]. An $M^{*}$-group $G$ is generated by three distinct nontrivial elements $t, u$, and $v$ which satisfy the relations

$$
t^{2}=u^{2}=v^{2}=(t u)^{2}=(t v)^{3}=1 .
$$

In particular, $G$ is generated by elements of order two. Suppose $G$ were nilpotent. Then these generators must belong to the normal Sylow 2-subgroup of $G$. Hence this subgroup would coincide with $G$ so that $G$ would be a 2 -group and its order could not be $12(g-1)$. Thus an $M^{*}$-group is not nilpotent. This observation combined with Theorem $\mathrm{A}$ yields our main result.

THEOREM 1. Let $G$ be a group of automorphisms of a bordered Klein surface of genus $g \geqslant 2$. If $G$ is nilpotent, then $o(G) \leqslant 8(g-1)$.

4. Examples. First we give several examples of surfaces of low genus with large nilpotent automorphism groups. Each example has a nice geometric flavor.

EXAMPLE 1. Let $\Lambda$ be the lattice subgroup of $C$ generated by $\{1, i\}$. Let $T$ be the torus $C / \Lambda$ and $\pi: C \rightarrow T$ the quotient map. Then rotation $90^{\circ}$ counterclockwise about the origin and reflection across the $x$-axis induce automorphisms of $T$ that generate a group $G \cong D_{4}$. The point $\pi(0)$ is clearly a fixed point of $G$. By removing from $T$ a disc centered at $\pi(0)$ we obtain a bordered surface $X$ of genus $g=2$ on which $G$ acts. Thus $X$ has a nilpotent group of automorphisms of order $8=8(g-1)$.

A similar example of the $M^{*}$-group $D_{6} \cong C_{2} \times S_{3}$ acting on a torus with one hole is in [2, p. 269].

EXAmple 2. Let $T$ be the torus of Example 1 . Translation by $(1+i) / 2$ also induces an automorphism $\alpha$ of the torus $T$. Then $\alpha$ is an involution that commutes with each element of $G$. Thus $T$ has an automorphism group $H \cong C_{2} \times G \cong C_{2} \times$ $D_{4}$. The group $G$ fixes the point $y=\pi((1+i) / 2)$, and $\alpha$ interchanges $\pi(0)$ and $y$. Now remove from $T$ discs centered about these two points. Then $H$ acts on the resulting bordered surface $Y$, a torus with two holes. Thus $Y$ is a surface of genus $g=3$ with a nilpotent automorphism group of order $16=8(g-1)$. 
EXAmple 3. Let $Z$ be a sphere with four holes, with the holes placed around the equator, centered at the vertices of an inscribed square. Then $Z$ has genus 3 . The surface $Z$ has a group of orientation-preserving automorphisms isomorphic to $D_{4}$, and reflection in the plane of the equator is an orientation-reversing automorphism. Hence $Z$ is another bordered surface of genus 3 with a nilpotent automorphism group of order 16. Again the group is isomorphic to $C_{2} \times D_{4}$.

EXAmple 4. Let $Z$ be the Klein surface of the previous example, and let $\tau$ : $Z \rightarrow Z$ be the antipodal map. The quotient space $W=Z / \tau$ is a real projective plane with two holes, a nonorientable Klein surface of genus $g=2$. Since each orientation-preserving automorphism of $Z$ commutes with $\tau, W$ has an automorphism group of order 8 isomorphic to $D_{4}$.

The actions of $D_{4}$ on the surfaces of genus 2 of Examples 1 and 4 were presented in [1] in a quite different way. The approach there utilizes noneuclidean crystallographic groups.

Now we see that both orientable and nonorientable bordered surfaces can have nilpotent automorphism groups of the maximum possible order. Next we apply constructions from [2] to find infinite families of surfaces for which the bound $8(g-1)$ is attained.

The theory of covering spaces and the fundamental group was used in $[2, \S 4]$ to construct full coverings of bordered Klein surfaces. Let $X$ be a bordered surface, and suppose a full cover $Z$ has been obtained by one of the constructions of [2, §4]. The construction gives the group $N$ of covering transformations and enough information to calculate the topological type of $Z$ from that of $X$. Since $Z$ is a full covering, each automorphism of $X$ lifts to an automorphism of $Z$. If we let $G=A(X)$ and $G^{*}$ be the group of automorphisms of $Z$ generated by $N$ and lifts of elements of $G$, then we have the exact sequence

$$
1 \rightarrow N \rightarrow G^{*} \rightarrow G \rightarrow 1 .
$$

Of course, $G^{*}$ is only a subgroup of $A(Z)$ in general.

EXAMPLE 5. Let $X$ be the torus with one hole of Example 1. Then for each positive integer $n$ there is a full cover $S_{n}(X)$ of $X$ with covering group $C_{n} \times C_{n}$ [2, Theorem 8, p. 273]. The surface $S_{n}(X)$ has genus $g_{n}=n^{2}+1$ and topologically is a torus with $n^{2}$ holes. The surface has an automorphism group $H_{n}$ of order $8 n^{2}$, since $A(X) \cong D_{4}$. Now let $n$ be a power of 2 . Then as a 2-group, $H_{n}$ is nilpotent, and $o\left(H_{n}\right)=8\left(g_{n}-1\right)$.

EXAmple 6. Let $Y$ be the torus with two holes of Example 2. Then for each positive integer $n$ there is a full cover $R_{n}(Y)$ with covering group $C_{n} \times C_{n}$ [2, Theorem 10, p. 274]. The surface $R_{n}(Y)$ has genus $\gamma_{n}=2 n^{2}+1$ and topologically is a torus with $2 n^{2}$ holes. Since $A(Y) \cong C_{2} \times D_{4}$, the surface $R_{n}(Y)$ has an automorphism group of order $16 n^{2}=8\left(\gamma_{n}-1\right)$. Again taking $n$ to be a power of 2 insures that the automorphism group is nilpotent.

Combining the last two examples yields the following result.

THEOREM 2. For each nonnegative integer $r$ there is a bordered Klein surface of genus $g=2^{r}+1$ that has a nilpotent group of automorphisms of order $8(g-1)$. 
Thus the bound $8(g-1)$ is attained for an infinite family of tori with holes. This family of bordered surfaces could be constructed in a more direct and geometric way from the torus $T$ of Example 1. In this connection see [2, pp. 269, 270].

The constructions of [2] can be used to produce many other topological types of bordered surfaces that have a nilpotent automorphism group of maximum possible order. Using a surface from one of our examples, we only need to construct a covering with degree a power of 2 . Then the automorphism group of the covering space will also be a 2-group. In particular, we could start with any surface from the infinite family, so that there is no shortage of examples. Interestingly, though, all the examples produced are orientable (even when the constructions are applied to the surface of Example 4).

5. Nilpotent groups of order $8(g-1)$. Each example of a nilpotent automorphism group of order $8(g-1)$ has been a 2 -group. Now we show that this type of group is the only one possible.

THEOREM 3. Let $G$ be a nilpotent group of automorphisms of the bordered Klein surface $X$ of genus $g \geqslant 2$. If $o(G)=8(g-1)$, then $g-1$ is a power of 2 .

Proof. By Theorem A the quotient space $X / G$ is the disc $D$. Further, the quotient map $\pi$ is ramified above four points of $D$, and each ramification index is 2 or 4.

Let $N$ be the Sylow 2-subgroup of $G$. Since $G$ is nilpotent, $N$ is normal in $G$. Then the order of the quotient group $G / N$ is not divisible by 2 . But $G / N$ acts on the quotient space $X / N$ with $(X / N) /(G / N)=X / G=D$, and the following diagram of quotient maps commutes:

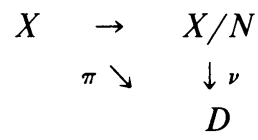

If $\nu$ is ramified above a point of $D$, then clearly so is $\pi$. Thus $\nu$ is ramified over at most four points of $D$, and the only possible ramification indices are 2 and 4 . But each ramification index of $\nu$ must divide $o(G / N)$. Hence the quotient map $\nu$ is an unramified covering of the disc $D$. Since $D$ has genus zero, the covering $\nu$ must be trivial, just from (2.1). Thus $N=G$ and $G$ is a 2-group.

A bordered Klein surface of genus $g \geqslant 2$ can easily have an automorphism group of order $8(g-1)$ that is not nilpotent. For example, let $W$ be a sphere with 8 holes, with the holes centered at the vertices of an inscribed cube. Then $W$ is a surface of genus 7 with an automorphism group isomorphic to $C_{2} \times S_{4}$, the complete symmetry group of the cube.

Suppose the nilpotent group $G$ acts on the bordered surface $X$ of genus $g \geqslant 2$. An obvious consequence of Theorems 1 and 3 is that if $G$ is not a 2-group, then the order of $G$ is strictly less than $8(g-1)$. In particular, this must be the case if $G$ is a $p$-group for some odd prime $p$. This raises the following natural problem (also, see [8, pp. 254, 255]). 
Problem 1. Let $p$ be an odd prime and $G$ a $p$-group of automorphisms of a bordered Klein surface of genus $g \geqslant 2$. Determine the best possible upper bound for $o(G)$.

There also still remains the interesting problem raised at the end of $\S 4$.

Problem 2. Let $G$ be a 2-group of automorphisms of a nonorientable bordered Klein surface of genus $g \geqslant 3$. Determine whether the bound $8(g-1)$ for $o(G)$ can be improved.

One good approach to this problem is to adapt a construction of Singerman in [6, §2]. Start with a noneuclidean crystallographic group $\Gamma$ generated by reflections $c_{1}$, $c_{2}$, and $c_{3}$ in the sides of a hyperbolic triangle with angles $\pi / 2, \pi / 4$, and $\pi / q$, where $q$ is a power of 2 and $q \geqslant 8$. Then $\Gamma$ has the presentation

$$
\Gamma=\left\langle c_{1}, c_{2}, c_{3} \mid c_{1}^{2}=c_{2}^{2}=c_{3}^{2}=\left(c_{1} c_{2}\right)^{2}=\left(c_{2} c_{3}\right)^{4}=\left(c_{1} c_{3}\right)^{q}=1\right\rangle .
$$

Let $\Gamma^{+}=\left\langle c_{1} c_{2}, c_{2} c_{3}\right\rangle$ be the subgroup of orientation-preserving isometries. Now let $G$ be a finite 2-group, and suppose that there is a homomorphism $\theta: \Gamma \rightarrow G$ preserving the orders of elements of finite order in $\Gamma$ such that $\theta\left(\Gamma^{+}\right)=G$. Then $G$ acts on a nonorientable bordered surface of genus $g$ such that $o(G)=8(g-1)$. We conjecture that such examples do exist, perhaps even for $g=5$.

Finally, we would like to thank the referee for an improvement to $\S 3$ and especially for pointing out the approach to Problem 2.

\section{REFERENCES}

1. E. Bujalance and J. M. Gamboa, Automorphism groups of algebraic curves of $R^{n}$ of genus 2 , Arch. Math. 42 (1984), 229-237.

2. N. Greenleaf and C. L. May, Bordered Klein surfaces with maximal symmetry, Trans. Amer. Math. Soc. 274 (1982), 265-283.

3. C. L. May, Automorphisms of compact Klein surfaces with boundary, Pacific J. Math. 59 (1975), $199-210$

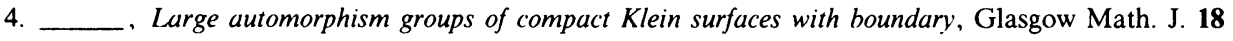
(1977), 1-10.

5. Supersolvable $M^{*}$-groups, Glasgow Math. J. (to appear).

6. D. Singerman, Orientable and non-orientable Klein surfaces with maximal symmetry, Glasgow Math. J. 26 (1985), 31-34.

7. $\operatorname{PSL}(2, q)$ as an image of the extended modular group with applications to group actions on surfaces, Proc. Edinburgh Math. Soc. 30 (1987), 143-151.

8. R. Zomorrodian, Nilpotent automorphism groups of Riemann surfaces, Trans. Amer. Math. Soc. 288 (1985), 241-255.

Department of Mathematics, Towson State University, Baltimore, Maryland 21204 